\title{
ANTECEDENTES DE INVESTIGACIÓN EN EL TRATAMIENTO DIGITAL DE SEÑALES ECG
}

\footnotetext{
T a finalidad de este capítulo es ofrecer una mirada retrospectiva al deLsarrollo de algoritmos basados en el tratamiento digital de señales que contribuyen a mejorar el proceso de análisis de la señal ECG. En etapas iníciales, el primer logro de la electrocardiografía fue la asociación del comportamiento del corazón con formas de ondas eléctricas, permitiendo abrir la puerta a una nueva técnica de diagnóstico. Consecutivamente se incentiva a mejorar los sistemas de adquisición de señales ECG y se emprende la integración de los dispositivos electrónicos. Esta integración ha logrado mejorar la sensibilidad de los sistemas de adquisición de señales ECG y la conectividad con dispositivos que permiten la transmisión de datos. Por último, se crea la necesidad de incluir los aportes del tratamiento digital de señales, los cuales han permitido la eliminación de distorsiones, detección de patrones y la cuantificación automatizada de parámetros a partir de la señal ECG. A continuación se presenta una breve recopilación de logros globales, nacionales y los aportados por el Semillero de Investigación en Procesamiento Digital de Señales de la Universidad Santo Tomás, Colombia.
} 


\section{El campo de la adquisición}

En primera instancia, el estudio del comportamiento del corazón mediante la señal eléctrica ECG nace en el trabajo del médico Einthoven [1]. Gracias a este trabajo se propuso en el año 1911 el primer prototipo de máquina llamada electrocardiógrafo. Un diagrama de bloques que se aproxima a este primer prototipo se puede ver en la figura 3.

FIGURA 3.

DIAGRAMA DE BLOQUES DEL PROTOTIPO PROPUESTO POR EINTHOVEN

FUENTE: ELABORACIÓN PROPIA.

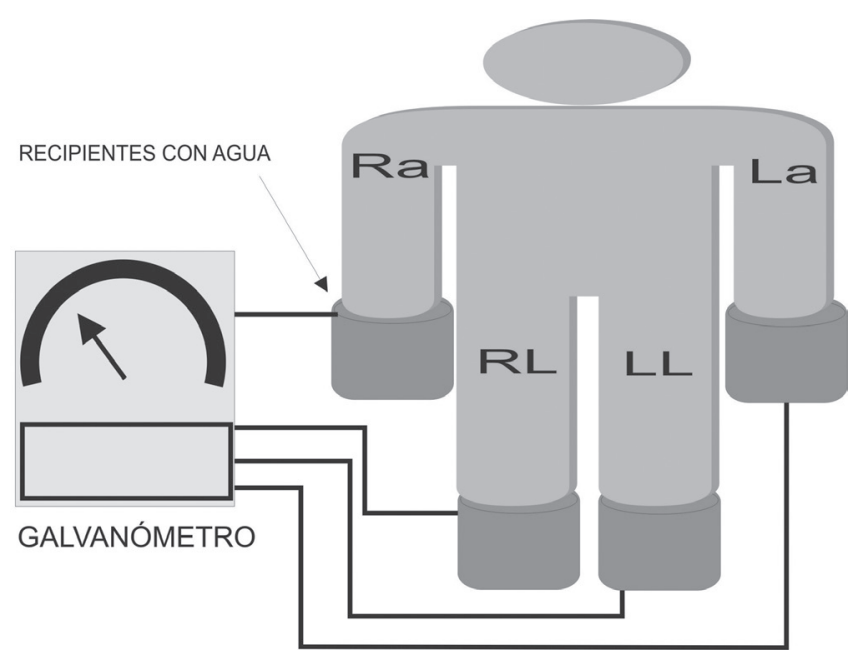

El prototipo de electrocardiógrafo, propuesto por Einthoven, no tiene originalmente electrodos adheridos a la superficie del sujeto. En la versión original, el electrocardiógrafo de Einthoven usa un conjunto de recipientes de agua en los cuales el paciente sumerge cada una de sus extremidades. Cada recipiente de agua tiene conectado un cable conductor que llega al sistema de registro basado en un galvanómetro, por lo cual esta configuración jugaba el papel de electrodo [1].

Con respecto a los electrodos, la tecnología ha ofrecido avances significativos que han permitido mejoras desde la propuesta de Einthoven. Los primeros modelos de electrodos constan de la combinación de un compuesto de plata y cloruro de plata $(\mathrm{Ag}-\mathrm{ClAg})$ y son desechables. Este estilo de electrodo es un elemento de reducido tamaño y se adhiere a la piel del sujeto por 
medio de un parche autoadhesivo. Por lo general, poseen una capa de una sustancia denominada gel dieléctrico y juega el papel de crear un acople de impedancia eléctrica entre la piel del sujeto y el electrodo. En la figura 4 se encuentra un ejemplo de modelo de electrodo [2].

\section{FIGURA 4. \\ MODELO DE \\ ELECTRODO \\ $A G-C L A G$}

FUENTE:

ELABORACIÓN

PROPIA.

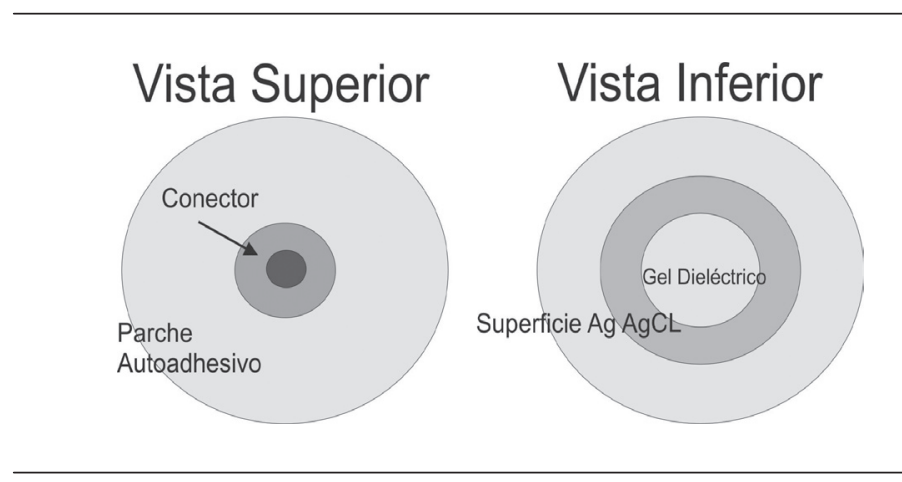

Entre las mejoras incluidas al diseño de electrodo se han introducido versiones capacitivas, que permiten un mejor acoplamiento a la piel y la reducción de interferencias [3]. Sin embargo, la investigación sigue ofreciendo nuevas tendencias en el tema de electrodos y la literatura ha reportado avances con nuevos modelos basados en carbón. Los electrodos a base de carbón permiten la reducción de irritaciones en la piel y la reutilización de los mismos [4]. Una ventaja adicional de los electrodos basados en carbón, es la posibilidad de ser operados en ambientes húmedos [5].

Otra característica importante de los electrodos es la adecuada ubicación espacial, que garantiza una buena adquisición de la señal ECG. La posición de los electrodos fue también propuesta por Einthoven, que tradicionalmente se colocan organizados en una configuración triangular que involucra las extremidades del sujeto. Actualmente se han dado aportes en nuevas formas de posicionar los electrodos utilizando configuraciones con menores cantidades de sensores [6].

El prototipo de electrocardiógrafo de Einthoven fue el inicio de una nueva era de sistemas para el diagnóstico a través del estudio de señales eléctricas. Gracias a esto, diversas empresas del sector del desarrollo de dispositivos electrónicos llevaron su mirada al desarrollo de sistemas orientados al sector 
salud. Un ejemplo de esto sucedió en el año 1928, cuando la empresa Frank Sanborn contribuyó al desarrollo de la tecnología para el estudio de esta señal eléctrica al producir el primer electrocardiógrafo portátil. Entre sus principales características estaba su peso $(25 \mathrm{Kg}$ ) y su fuente de energía que requería la alimentación de una batería de automóvil de 6 Voltios [7].

Los sistemas de adquisición de señales ECG, como el producido por la empresa Sanborn, tienen la limitación que solo pueden ser utilizados para exámenes en una condición estática. En consecuencia, no se podría estudiar como la señal ECG experimentaba cambios correlacionados con el comportamiento diario del sujeto bajo estudio. Estas necesidades de mejorar la tecnología para adquirir señales ECG, llevaron al desarrollo de prototipos con la capacidad de almacenamiento de registros con tiempos de 24 horas. Por tal razón, en 1949, el médico Norman Holter desarrolló un dispositivo para almacenar señales ECG. Actualmente este tipo de prototipos son utilizados para registros ambulatorios [8]. Un dispositivo tipo Holter debe estar compuesto de diferentes elementos que garanticen su portabilidad. En la figura 5 se puede encontrar un diagrama de bloques que contiene los elementos de un sistema para señales ECG tipo Holter.
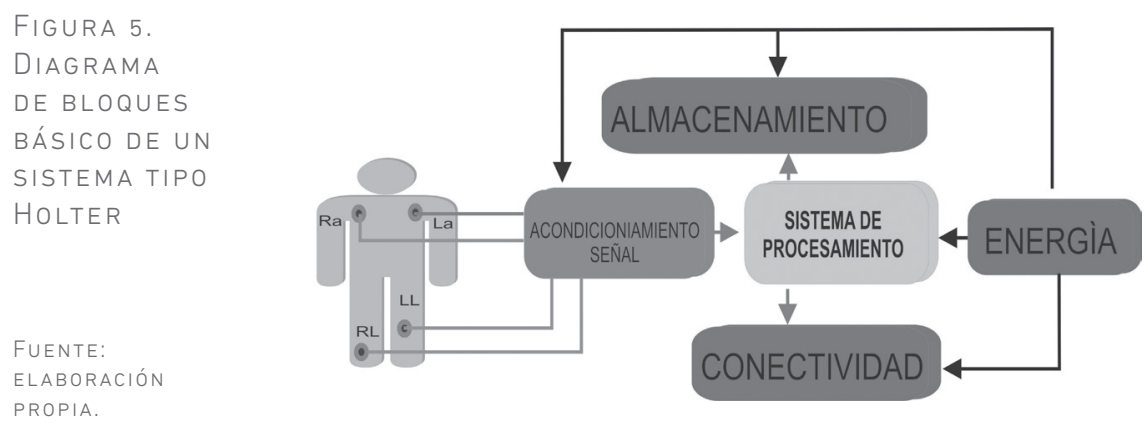

Con referencia a la figura 5, un sistema básico tipo Holter debe contener una fuente de energía, como por ejemplo una batería recargable. También debe contar con un sistema de almacenamiento de información o memoria, recomendablemente de estado sólido. Por último, este tipo de sistema 
requiere de un módulo de comunicación para poder descargar los datos a un computador personal. En la actualidad, un sistema tipo Holter es administrado por un sistema digital programable tipo microcontrolador o un dispositivo digital programable. El valor agregado de los sistemas Holter consiste en que es un dispositivo portátil que puede ser conectado al paciente y permitir la adquisición de señales ECG en situaciones dinámicas [9]

También se han desarrollado otras versiones de electrocardiógrafos, especialmente orientados al monitoreo fetal. Este tipo de monitoreo es más exigente, debido a la superposición del electrocardiograma de la madre sobre el de su feto. Este tipo de sistema requiere de la implementación de sistemas adaptativos para mejorar el análisis de la señal ECG [10]. Otros desarrollo tecnológicos se han orientado a nuevas formas de adquisición de la señal ECG, como es el caso de los sistemas de monitoreo del esófago. Este tipo de sistema permite la introducción de electrodos, a través del esófago del sujeto, para tener un acercamiento al corazón [11]. Como otra alternativa se han explorado técnicas de adquisición usando el posicionamiento de juegos de electrodos en bañeras, con la capacidad de adquirir señales ECG mediante la facilidad del medio acuoso [12].

Desde el punto de vista de conectividad, los sistemas de adquisición de señales ECG se han beneficiado gracias a la vinculación de dispositivos de transmisión de datos inalámbricos. En versiones anteriores de prototipos de sistemas de adquisición de señales ECG, se contaba con la limitación de contar con un medio físico rígido conectado a un computador personal. Este tipo de conexión es importante para garantizar un flujo de datos para almacenamiento, visualización y posterior análisis de la señal ECG. Para romper esta limitación se ha optado por el uso de sistemas de interface que permiten que los datos obtenidos puedan viajar a través del aire. Este tipo de interface está controlado por protocolos estándar para la recepción y transmisión de datos mediante señales electromagnéticas. Uno de los protocolos más utilizado es el bluetooth, el cual permite generar transmisiones de señales ECG con menor gasto de energía [13].

En el contexto colombiano, diversas instituciones de educación superior, orientadas a estudios clínicos, han desarrollado aportes en cuanto al tema de 
adquisición y tratamiento de señales ECG. En primera instancia se destaca el trabajo del ingeniero Jorge Reynolds, por sus aportes en innovar en técnicas de telemetría para adquisición de señales ECG de personas y también de mamíferos de gran tamaño sumergidos en agua [14].

En cuanto a la mejora de dispositivos para censar variables directamente del corazón y poder interactuar con este mismo órgano, el ingeniero Ignacio Escobar colaboró con el estado del arte, en cuanto al diseño de sistemas de instrumentación médica [15].

Diversos prototipos de sistemas portátiles para adquirir y almacenar señal ECG se han podido diseñar. Existen antecedentes de prototipos orientados al estudio de la variabilidad de la frecuencia cardíaca, que tienen la posibilidad de adquirir la señal ECG y la cuantificación de frecuencia instantánea [16].

\section{Con respecto a las técnicas de tratamiento y procesamiento de la Señal ECG}

Con el avance del diseño de sistemas para adquisición de señales ECG, surge la necesidad de impulsar nuevos métodos para atenuar las distorsiones. En cuyo caso se generan trabajos interesantes basados en el tratamiento digital de señales. Sobresalen las investigaciones del autor Tompkins Willis y sus primeros aportes en la aplicación de filtros digitales basados en ecuaciones en diferencia [17]. El estudio de Tompkins plantea las primeras metodologías en torno al diseño de filtros en microprocesadores para eliminación de los efectos de interferencias.

Además del uso de filtros digitales aplicados a la reducción de distorsiones, se suman las primeras aplicaciones para la detección de parámetros. Entre los primeros trabajos se encuentran los primeros algoritmos orientados a la detección de la onda $\mathrm{R}$ del complejo cardíaco. Se destacan las primeras aplicaciones de bancos de filtros, que consisten en un método iterativo para estimar el rango de frecuencias que contienen la información de la onda R [18].

Al mismo tiempo, en el contexto nacional se empiezan a emplear métodos de análisis de la señal ECG en el dominio de la frecuencia. Diferentes trabajos han involucrado la implementación de algoritmos basados en la transformada de Fourier. Las estrategias basadas en la transformada de 
Fourier permiten realizar la comparación de la señale ECG con diferentes señales sinusoidales con diversos valores en frecuencia. Estos aportes no solo permitieron avances en la detección de parámetros, sino también el desarrollo de las primeras aplicaciones de compresión de la señal ECG [19].

La señal ECG no solo puede ser estudiada a través de comparaciones con señales sinusoidales. Esto es debido a que muchos de sus parámetros poseen formas de ondas diferentes. Como alternativa, diversos autores consideran el uso de otras formas de análisis en el dominio de la frecuencia, como es el caso de la transformada Wavelet [20].

\section{El entorno nacional}

Con respecto al tema de mejoras en las técnicas de filtrado, se han realizado aportes relacionados con la aplicación de filtros adaptativos, orientados a eliminar las distorsiones causadas por el desplazamiento de línea de base y el ruido de las líneas de alimentación de energía eléctrica. En este tipo de trabajos, se verifica que las técnicas de filtrado digital convencional pueden eliminar interferencias pero también características importantes de la señal ECG [21], [22].

Con la finalidad de mejorar la sensibilidad de los algoritmos para tratar de encontrar más información contenida de la señal ECG, se han generado estrategias relacionadas con la electrocardiografía fetal. En este tipo de contribuciones se han tratado señales electrocardiográficas de mujeres en estado de embarazo, cuya señal ECG contiene la información del electrocardiograma fetal [23].

Los estudios sobre señal ECG no solo han sido utilizados para extraer información contenida en registros tomados de seres humanos. En el contexto colombiano, se han dado aportes basados en la aplicación de técnicas de filtrado digital y transformada de Fourier para el análisis de señal ECG de felinos. El estudio de señales ECG de otros mamíferos permite encontrar parámetros que pueden ser comparados con los encontrados en seres humanos y otras especies animales [24]. 


\section{El aporte del semillero de investigación}

El apoyo de la Universidad Santo Tomás a los grupos y semilleros de investigación ha generado aportes interesantes en cuanto al tratamiento y análisis de la señal ECG. Así, por ejemplo, en el año 2013, durante la convocatoria del Fondo de Investigaciones FODEIN, se aprobó el proyecto "Plataforma informática para el análisis de la variabilidad de la frecuencia cardíaca orientado a la prestación de servicios de análisis y cálculo de descriptores”. El proyecto en mención planteó el diseño y la implementación de un conjunto de algoritmos orientados al análisis de la señal ECG [25].

Dentro de los algoritmos generados en la plataforma informática, se desarrolló una estrategia basada en bancos de filtros. Esta estrategia permite evaluar el comportamiento de la señal ECG ante el filtrado de diferentes bandas de frecuencia. Como resultado se pudo obtener un criterio para establecer el ancho de banda de filtros digitales, en busca del realce de la onda $\mathrm{R}$ del complejo cardíaco [26].

Como aporte al cálculo se descriptores, es importante el desarrollo de técnicas para mejorar la información obtenida a partir de los parámetros extraídos de la señal ECG. Dentro de estos parámetros están los tiempos RR, que corresponden a las mediciones entre cada onda R de la señal ECG. Al interior del análisis de la variabilidad de la frecuencia cardíaca, es necesario el estudio del espectro de la serie de tiempo que contiene todos los valores de las mediciones entre cada onda R. El análisis espectral de la señal de tiempos RR puede poseer rizados y efectos pasa bajos, por lo cual se ha realizado un aporte para contrarrestar este tipo de sucesos [27].

De manera consecutiva, el Semillero de Investigación en Procesamiento Digital de Señales logró la aprobación del proyecto titulado: "Sistema digital para la cuantificación eficaz de la frecuencia cardíaca instantánea”. Dentro de este proyecto se pudo lograr el diseño e implementación de un filtro en tiempo real para el realce de la onda R de la señal ECG. En esta implementación se utiliza el criterio para determinación del ancho de banda que contiene las componentes espectrales de la onda R, con la finalidad de establecer las frecuencias de corte para un filtro digital. El sistema de filtrado fue implemen- 
tado en un dispositivo programable tipo ordenador con conjunto reducido de instrucciones (ARM) [28].

En este primer capítulo se ha resumido un panorama sobre los desarrollos tecnológicos que giran alrededor de la necesidad de estudiar el sistema cardiovascular. Muchos estudios de este sistema se han podido lograr gracias a la adquisición, tratamiento y procesamiento de la señal ECG. Cabe resaltar que, en un principio, solo fue importante relacionar una característica eléctrica con un proceso mecánico. Pero con el pasar de los años las exigencias de los medios de diagnósticos empezaron a requerir un esfuerzo en los aportes de los dispositivos electrónicos.

Actualmente los equipos dedicados al diagnóstico y monitoreo de personas, no solo deben conformarse con la adquisición de la señal ECG. También es de gran importancia la integración de la programación, las matemáticas y la implementación en sistemas digitales programables. Esta integración ha permitido la generación de valores agregados a los equipos de diagnóstico y monitoreo. 
